\title{
Long-Term Effects of Self-Administered Transcranial Direct Current Stimulation in Episodic Migraine Prevention: Results of a Randomized Controlled Trial
}

Pohl, Heiko ; Moisa, Marius ; Jung, Hans-H ; Brenner, Kathrin ; Aschmann, Jessica ; Riederer, Franz ; Ruff, Christian C ; Schoenen, Jean ; Luechinger, Roger ; Widmer, Lukas ; Petersen, Jens A ; Gantenbein, Andreas R ; Sandor, Peter S ; Michels, Lars

\begin{abstract}
BACKGROUND Migraine is a multifactorial neurovascular disorder, which affects about $12 \%$ of the general population. In episodic migraine, the visual cortex revealed abnormal processing, most likely due to decreased preactivation level. Transcranial direct current stimulation (tDCS) is able to modify cortical excitability and might result in an alleviation of migraine occurrence if used repetitively. OBJECTIVE To test the hypothesis that self-administered anodal tDCS over the visual cortex significantly decreases the number of monthly migraine days in episodic migraine. MATERIALS AND METHODS The study was single-blind, randomized, and sham-controlled. Inclusion criteria were age 18-80 years and an ICHD-3 diagnosis of episodic migraine. Exclusion criteria were pregnancy, presence of a neurodegenerative disorder, a contraindication against MRI examinations, and less than two migraine days during the 28-day baseline period. Patients in whom the baseline period suggested chronic migraine were excluded. After baseline, participants applied daily either verum (anodal-1 mA to $20 \mathrm{~min}$ ) or sham tDCS (anodal-1 mA to $30 \mathrm{sec}$ ) at $\mathrm{Oz}$ (reference $\mathrm{Cz}$ electrode) for 28 days. Headache diaries were used to record the number of migraine days at baseline, during the stimulation period, and during four subsequent 28-day periods. RESULTS Twenty-eight patients were included; two were excluded after the baseline period because less than two migraine days occurred; three were excluded because their headache diaries suggested the diagnosis of chronic migraine. Twenty-three datasets were taken for further analysis. Compared to sham tDCS $(\mathrm{n}=12)$, verum tDCS $(\mathrm{n}=11)$ resulted in a lower number of migraine days $(\mathrm{p}=0.010)$ across all follow-up periods. We found no significant change in total headache days $(\mathrm{p}=$ $0.165)$, anxiety $(\mathrm{p}=0.884)$, or depression scores $(\mathrm{p}=0.535)$. No serious adverse events occurred; minor side effects were similar in both groups. CONCLUSIONS This study provides Class II evidence that self-administered anodal tDCS over the visual cortex in episodic migraine results in a significantly lower number of monthly migraine days. However, it has neither an immediate nor a long-term effect.
\end{abstract}

DOI: https://doi.org/10.1111/ner.13292

Posted at the Zurich Open Repository and Archive, University of Zurich

ZORA URL: https://doi.org/10.5167/uzh-191495

Journal Article

Accepted Version

Originally published at:

Pohl, Heiko; Moisa, Marius; Jung, Hans-H; Brenner, Kathrin; Aschmann, Jessica; Riederer, Franz; Ruff, Christian C; Schoenen, Jean; Luechinger, Roger; Widmer, Lukas; Petersen, Jens A; Gantenbein, 
Andreas R; Sandor, Peter S; Michels, Lars (2021). Long-Term Effects of Self-Administered Transcranial Direct Current Stimulation in Episodic Migraine Prevention: Results of a Randomized Controlled Trial. Neuromodulation, 24(5):890-898.

DOI: https://doi.org/10.1111/ner.13292 


\section{Long-term effects of self-administered tDCS in episodic migraine prevention: results of a randomized controlled trial}

Heiko Pohl ${ }^{1}$ (ORCID 0000-0002-2778-6790), Marius Moisa² (ORCID 0000-0001-9789-3383), Hans-H. Jung ${ }^{1}$, Kathrin Brenner $^{3}$, Jessica Aschmann ${ }^{3}$, Franz Riederer ${ }^{1,4}$ (ORCID 0000-0002-9722-9754), C.C. Ruff ${ }^{2}$ (ORCID 0000-0002-39642364), Jean Schoenen ${ }^{5}$ (ORCID 0000-0002-6506-6460), Roger Luechinger (ORCID 0000-0002-7365-0421) , Lukas Widmer $^{1}$, Jens A. Petersen (ORCID 0000-0003-0777-4263) ${ }^{7}$, Andreas R. Gantenbein ${ }^{1,8}$, Peter S. Sandor ${ }^{1,8}$, Lars Michels (ORCID 0000-0003-3750-1100)

\section{Affiliation}

${ }^{1}$ Department of Neurology, University Hospital Zurich, Zurich, Switzerland

${ }^{2}$ Zurich Center for Neuroeconomics (ZNE), Department of Economics, University of Zurich, Zurich, Switzerland

${ }^{3}$ Department of Neuroradiology, University Hospital Zurich, Zurich, Switzerland

${ }^{4}$ Neurological Center Rosenhügel and Karl Landsteiner Institute for Epilepsy Research and Cognitive Neurology, Vienna, Austria

${ }^{5}$ Headache Research Unit, Department of Neurology-Citadelle Hospital, University of Liège, Liège, Belgium

${ }^{6}$ Institute for Biomedical Engineering, ETH Zurich and University of Zurich, Zurich, Switzerland

7 Neurozentrum Bern, Bern, Switzerland

${ }^{8}$ RehaClinic Bad Zurzach, Bad Zurzach, Switzerland

\section{Corresponding Author}

Lars Michels, Department of Neuroradiology, University Hospital Zurich, Zurich, Switzerland; telephone +41 44255 4965, email address lars.michels@usz.ch

Statistical Analyses were conducted by Heiko Pohl, MD, University Hospital Zurich, Switzerland

Word count

- Main Article: 4182

- Abstract: 306

- Title (character count): 117

\section{Abbreviations}

$\begin{array}{ll}\text { CM } & \text { chronic migraine } \\ \text { EM } & \text { episodic migraine } \\ \text { FUP1 } & \text { first follow-up visit } \\ \text { FUP2 } & \text { second follow-up visit } \\ \text { HADS } & \text { Hospital Anxiety and Depression Scale } \\ \text { ICHD-3 } & \text { International Classification of Headache Disorders, 3rd edition } \\ \text { MIDAS } & \text { migraine disability assessment } \\ \text { tDCS } & \text { transcranial direct current stimulation }\end{array}$




\section{Search Terms}

tDCS, migraine, neurostimulation, neuromodulation, hyperexcitability, migraine days 


\section{Abstract}

Background: Migraine is a multifactorial neurovascular disorder which affects about $12 \%$ of the general population. In episodic migraine, the visual cortex revealed abnormal processing, most likely due to decreased pre-activation level. Transcranial direct current stimulation (tDCS) is able to modify cortical excitability and might result in an alleviation of migraine occurrence if used repetitively.

Objective: To test the hypothesis that self-administered anodal tDCS over the visual cortex significantly decreases the number of monthly migraine days in episodic migraine.

Methods: The study was single-blind, randomised, and sham-controlled. Inclusion criteria were age 1880 years and an ICHD-3 diagnosis of episodic migraine. Exclusion criteria were pregnancy, presence of a neurodegenerative disorder, a contraindication against MRI examinations, and less than two migraine days during the 28-day baseline period. Patients in whom the baseline period suggested chronic migraine were excluded. After baseline, participants applied daily either verum (anodal-1mA-20min) or sham tDCS (anodal-1mA-30sec) at Oz (reference Cz electrode) for 28 days. Headache diaries were used to record the number of migraine days at baseline, during the stimulation period, and during four subsequent 28-day periods.

Results: Twenty-eight patients were included; two were excluded after the baseline period because less than two migraine days occurred; three were excluded because their headache diaries suggested the diagnosis of chronic migraine. Twenty-three datasets were taken for further analysis. Compared to sham $\operatorname{tDCS}(n=12)$, verum tDCS $(n=11)$ resulted in a lower number of migraine days $(P=0.010)$ across all followup periods. We found no significant change in total headache days $(P=0.165)$, anxiety $(P=0.884)$, or depression scores $(P=0.535)$. No serious adverse events occurred; minor side effects were similar in both groups.

Conclusions: This study provides Class II evidence that self-administered anodal tDCS over the visual cortex in episodic migraine results in a significantly lower number of monthly migraine days. However, it has neither an immediate nor a long-term effect. 


\section{Introduction}

Brains of migraine patients process sensory information differently(1). Photo-, and Phonophobia are common both during $(2,3)$ and between $\operatorname{attacks}(4,5)$. Interictally, many migraineurs do not habituate to repeated somatosensory(6), visual(7), acoustic(8), and painful stimuli(9).

Several studies investigated the cortical responsivity in order to elucidate underlying mechanisms. In episodic migraine (EM), the abnormal processing in the visual cortex is thought to reflect a decreased pre-activation level due to an insufficient thalamo-cortical drive(10, 11). In chronic migraine (CM), however, some studies indicate hyperexcitability of visual and somatosensory cortices(12, 13). Even though not all studies confirm these findings and alternative interpretations have been proposed(14), there is evidence for altered cortical information processing over the migraine cycle.

Transcranial direct current stimulation (tDCS) can modify cortical excitability(15-18) presumably through hyperpolarisation or subthreshold depolarisation of neurones depending on current direction(19). Consequently, tDCS seems to lend itself to the treatment of functional disorders characterised by altered brain excitability, as present in migraine. This appears all the more attractive since this approach has no serious side effects, while most preventive migraine pharmacotherapies have an unfavourable efficacy/adverse effect profile(20).

The suitability of tDCS for the treatment of migraine was examined in several studies applying tDCS to the occipital cortex(21-24), the primary motor cortex(25-27), or the dorsolateral prefrontal cortex(26, 28) of migraine patients. In most - not all(23) - of these open-label $(21,27,28)$ and sham-controlled trials $(22,24)$, a significant reduction of migraine days was achieved. In addition, tDCS restored habituation to repeated visual stimuli in patients with $\operatorname{EM}(21,29)$.

While in some studies anodal stimulation was used(21, 25-27), in others cathodal stimulation was chosen(22-24), and one study combined both approaches(28). The difference between these techniques is noteworthy as anodal stimulation is generally viewed as increasing and cathodal stimulation as decreasing cortical excitability(16-18), although not everybody responds in the predicted $\operatorname{way}(30,31)$.

Considering the cited findings, one may speculate that anodal tDCS is best suited for EM and cathodal stimulation for CM, as these disorders may be characterised by cortical hypo- and hyperexcitability, respectively(10-13). This hypothesis has not yet been confirmed and not in every study undertaken so far, EM and CM were distinguished.

In this study, we decided to tailor the stimulation pattern to the supposed cortical pre-activation level of the participants' visual cortices by treating EM patients with (presumably activating) anodal tDCS over the occipital cortex. We analysed the effect of a 28-day treatment period on migraine/headache days and disability, but also on anxiety and depression that are often comorbid with migraine(32).

We also decided to study self-administered tDCS, which may be challenging. Laypersons who mount electrodes themselves might indeed be unable to position them correctly despite adequate training and thereby impede correct stimulation, since minor deviations of the electrode position can impact both the current path and the induced electric field(33,34), and may prevent the electric field from concentrating under the active electrode(35).

In addition, we assessed the persistence of the stimulation effect up to four months post-treatment. So far, most studies did not evaluate the duration of the clinical tDCS effects in migraine, except for one study that found a significant reduction in migraine attacks up to eight weeks after the end of the stimulation(36). 
We hypothesized that, compared to sham stimulation, anodal tDCS would result in a long-lasting reduction of migraine days in patients suffering from EM. To our knowledge, no randomised, sham controlled study (with a longitudinal design) has yet addressed this research question. Thus, the novelties of this tDCS study in episodic migraine prevention are application of an excitatory protocol over the visual cortex, self-administration by the patients and investigation of long-term effects.

\section{Methods}

\section{Participants and inclusion/exclusion criteria}

All participants gave their informed consent and the local ethics committee approved the study. Inclusion criteria were age between 18 and 80 years and a diagnosis of "EM without aura" or of "EM with and without aura" according to the ICHD-3 criteria(3). All headache diagnoses had been made by a neurologist.

Exclusion criteria were pregnancy, presence of a neurodegenerative disorder, and contraindication against an MRI examination. Patients who had less than two migraine days during the 28-day baseline period were excluded. In addition, we validated the diagnosis of EM based on the baseline period; participants who had 15 or more headache days and eight or more migraine days were excluded because these symptoms were more consistent with a diagnosis of $\mathrm{CM}$.

\section{Headache diary}

Participants kept headache diaries(37) throughout the study and recorded occurrence, duration, quality and intensity of headache attacks, as well as acute medication intake and accompanying features.

Migraine days were identified and distinguished from days with other headache types based on the headache diary using the criteria proposed by Tassorelli and co-workers(38). A migraine day was defined as day with a headache that lasts at least four hours and fulfils one of the conditions. (i) Criteria $\mathrm{C}$ and D for migraine without aura according to the ICHD-3 are met; (ii) criteria B and C for migraine with aura are met, (iii) criteria for probable migraine are met; (iv) the headache is treated successfully with a triptan. We defined moderate to severe pain as pain ratings of four and above on a numerical rating scale ranging from zero to 10 , zero indicating the absence of pain and 10 the strongest imaginable pain.

Completeness of the headache diaries was evaluated on a monthly basis. If headache days had been recorded incompletely, data for that period were considered missing; we did not impute.

\section{Study design}

This monocentric, single-blind, randomised, and sham-controlled trial was conducted at the University Hospital Zurich. Sample size was not calculated before the study, because no pilot data were available for the chosen study design. Participants were randomised to receive either verum or sham tDCS; group allocation was concealed from all participants and the principal investigator until the end of the study. We used a block randomisation technique with block sizes of ten (comprising 5 verum and 5 sham per block). Two authors, KB and JA enrolled and assigned participants to interventions; they also had generated the random allocation sequence.

Throughout the study period, participants recorded headache days in calendars. To allow statistical analysis, we subdivided the diary entries into six subsequent 28-day periods, which will be referred to as baseline period, $T 1, T 2, T 3, T 4$, and T5. The prospective 28-day baseline period ended with the baseline visit. The next day, the 28-day stimulation period (referred to as T1) was launched. After that, patients recorded their headaches during four subsequent 28-days periods (T2, T3, T4, and T5; see figure 1). 
In addition, all participants were examined in three presential visits: baseline visit, follow-up visit 1 (FUP1), and follow-up visit 2 (FUP2); see figure 1. The baseline visit marked the end of the baseline period and the onset of T1; the FUP 1 was scheduled shortly after the stimulation period (T1) and FUP2 took place after T5, about six months post-baseline. At each of these visits, multiparametric MRI examinations were performed; these data will be published separately. The trial was registered at ClinicalTrials.gov (Identifier: NCT03237754).

\section{tDCS}

tDCS was performed using a one-channel stimulator and standard rubber tDCS electrodes provided by the manufacturer (DC-STIMULATOR PLUS, NeuroConn, IImenau, Germany). Participants applied anodal stimulation over the visual cortex (see Figure $2 \mathrm{~A}$ for the tDCS montage). The active electrode was placed at $\mathrm{Oz}$ (electrode size $5^{*} 7 \mathrm{~cm}^{2}$ ), while the reference electrode was placed at $\mathrm{Cz}$ (electrode size 10*10 $\mathrm{cm}^{2}$ ). $\mathrm{Oz}$ is located at the inion, while the $\mathrm{Cz}$ is located at the intersection between a sagittal line from the nasion to the inion and a coronal line connecting the tragus of both ears. We chose a more focal electrode to maximize the current density over the visual cortex (current density of the active electrode is $0.029 \mathrm{~mA} / \mathrm{cm}^{2}$ ) and a large reference electrode to minimize current density at the $\mathrm{Cz}$ area (current density of the reference electrode is $0.01 \mathrm{~mA} / \mathrm{cm}^{2}$ ). Thus, the large reference electrode was functionally ineffective(39).

At the baseline visit the investigator explained the electrode placement to the participants and instructed them to first place the rubber electrodes in the sponge pads (NeuroConn, Ilmenau, Germany) and to soak them in saline water. The electrodes were kept in place by standard elastic rubber straps.

tDCS was performed at a $1 \mathrm{~mA}$ intensity for 20 minutes per session. We chose $1 \mathrm{~mA}$ in order to minimize the possible discomfort of the patients during the stimulation, and encourage future studies comparing the effects of $1 \mathrm{~mA}$ and $2 \mathrm{~mA}$. Sham stimulation also had $1 \mathrm{~mA}$ intensity but was maintained for only 30 seconds; during the remaining 1170 seconds only intermittent impedance checks occurred. We computed the normalized electric field distribution using a realistic finite element head model(40) implemented in the Simnibs 2.1 toolbox (https://simnibs.github.io/simnibs)(41). As expected, the predicted electric field is strongest in the vicinity of the active electrode placed over the visual cortex (see Figure 2 B). Participants applied verum or sham tDCS at home once daily over a four-week period and logged sessions in a stimulation diary.

\section{Efficacy endpoints}

The primary endpoint was the overall estimated marginal mean change from baseline in the number of monthly migraine days across T2 to T5. A secondary endpoint was the overall marginal mean change from baseline in the number of monthly headache days, medication days, migraine intensity, and headache intensity across $\mathrm{T} 2$ to $\mathrm{T} 5$.

Further secondary endpoints were the estimated marginal mean changes from baseline in monthly migraine days during $\mathrm{T} 1, \mathrm{~T} 2, \mathrm{~T} 3, \mathrm{~T} 4$, and $\mathrm{T} 5$. In addition, we calculated change from baseline in monthly headache and medication days as well as in average intensity of migraine and headache days during T4; we decided to calculate differences between sham and anodal stimulation during T4 as between-group differences were largest during that period (see results). Finally, we calculated the percentage of patients whose number of monthly migraine and headache days was halved in T4 compared to baseline (50\%-responder rate).

Further secondary endpoints were changes from baseline in the score of the hospital anxiety and depression scale (HADS-A and HADS-D, respectively) as well as the migraine disability assessment scale (MIDAS) at the first and second follow-up visit (FUP1 and FUP2). 


\section{Safety}

All participants were asked to record side effects of the intervention. In particular, we asked for tingling, pain, nausea, and fatigue. Adverse events that occurred during and up to four weeks after the stimulation period were registered (in paper form) as well. No additional exams (e.g. vital signs, neurological examination, etc.) were performed at the follow-up visits.

\section{Statistical analysis}

The analyses were conducted in the modified intention-to-treat population, which consisted of all participants who had completed the stimulation period (T1).

We analysed the primary endpoint -i.e., the overall mean change from baseline in the monthly number of migraine days - using a mixed effects model. Categorical fixed effects were group assignment (anodal versus sham stimulation). Continuous fixed effects were the baseline frequency of migraine days, the number of stimulation days, the score in the HADS-D, as well as the stimulation days-by-baseline migraine frequency interaction. We included the depression score as covariate because there is evidence of depression altering cortical excitability(42-45). Normal distribution of dependent variables was assessed before analysis using the Shapiro Wilk test.

Some participants applied tDCS for more or less than 28 days. To account for this imbalance, we included the number of stimulation days as covariate into the statistical model. To prevent inconsistencies caused by different lengths of $\mathrm{T} 1$, the reported frequencies were normalised to migraine and headache days per 28 days.

In order to track changes in migraine frequency over the study period, we counted migraine days during baseline as well as T1, T2, T3, T4, and T5 and estimated marginal changes separately for each period, by conducting a mixed effect model for each period analogously to the above-mentioned approach.

Secondary endpoints analysing changes from baseline in total headache days and medication days as well as changes in intensity in T4 were analysed analogously to the primary endpoint. Instead of the baseline number of migraine days, baseline values of the relative variable were added as continuous fixed effect.

Likewise, we assessed changes in the average score in the HADS-A, HADS-D, and MIDAS using mixed effects models. Since the follow-up visits were postponed in some cases, we included the length of the delays as covariate into the mixed effect model.

Serial testing served as gatekeeping strategy to prevent the increase of the overall type I error by multiple testing(46). Statistical testing of the secondary endpoints was not pursued if the stimulation effect analysed in the primary endpoint did not have a significant impact on migraine days. We did not correct for multiple testing; thus, the analyses of the secondary endpoints need to be considered exploratory.

Group differences were assessed with two-sided t-tests, ANOVA, and Chi-squared test. We used IBM SPSS statistics version 25 for the calculations; significance level was set at 0.05 .

\section{Classification of evidence}

We used the AAN Classification of Evidence Matrix to determine the class of evidence(47). The study was designed to answer the following research question: Does tDCS using anodal stimulation for EM influence the number of monthly migraine days?

Data availability

The data collected and analysed for the current study are available from the corresponding author on reasonable request. 


\section{Results}

We screened 28 participants with EM. Two patients were excluded after the baseline period because less than two migraine days occurred; three patients were excluded because they had recorded in their headache diaries 15 or more headache days and eight or more migraine days during the baseline period and were thus more likely to suffer from CM than EM. The trial was halted due to recruitment difficulties. The characteristics of the remaining 23 participants are listed in Table 1. Fourteen participants had reported at least one aura during the study period $(14 / 23,61 \%)$; of these seven (50\%) had received sham tDCS stimulation. One patient did not complete the headache diary during T2; apart from that, there were no missing data.

While we planned a stimulation period of 28 days, some patients used their stimulation device for less or more than 28 days (on average $29 \pm 2$ days). The average number of stimulation days was higher in the tDCS groups than in the sham group (tDCS $30 \pm 3$ days, sham $28 \pm 0.5$ days; $P=0.044$ ).

\section{Efficacy endpoints}

Across all patients, the estimated marginal mean change in monthly migraine days across T2 to T5 was $-1.7 \pm 0.5$ days $(95 \%-\mathrm{Cl}-2.6--0.7)$ in the anodal tDCS group and $0.2 \pm 0.4$ days $(95 \%-\mathrm{Cl}-0.7-1.1)$ in the sham tDCS group; a positive number implies an increase in migraine days. The overall between-group difference across T2 to T5 was $1.9 \pm 0.7$ days ( $95 \%$ confidence interval: $0.5-3.3 ; \mathrm{P}=0.010$ ). Changes from baseline in migraine days were normally distributed in all periods $T 2$ to $T 5$ according to the Shapiro Wilk test $(\mathrm{P}=0.497, \mathrm{P}=0.633, \mathrm{P}=0.439$, and $\mathrm{P}=0.682$, respectively). Thus, our data show that anodal $\operatorname{tDCS}$ over the visual cortex significantly decreases the number of migraine days across a 5 -month period. When adding a factor to the model that differentiates participants who had at least one aura during the study period from those without an aura, this variable was not found to have a significant influence on changes in migraine days $(\mathrm{P}=0.114)$.

Considering each period ( $\mathrm{T} 1$ to $\mathrm{T} 5)$ separately, changes in migraine days at $\mathrm{T} 4$ ( $\mathrm{P}=0.033)$ were significant and there was a trend for significance at $\mathrm{T} 3(\mathrm{P}=0.081)$. However, there was no significant difference in changes between the two groups during stimulation ( $T 1 ; P=0.944)$, as well as in T2 ( $P=0.497)$, and T5 $(P=0.631)$; see Figure 3 for graphical depiction.

During T4, four patients in the tDCS group (4/11,36.3\%) and two patients in the sham group (2/12, $16.7 \%$ ) had a reduction of at least $50 \%$ in the number of migraine days (50\%-responders); the difference was not significant $(P=0.371)$.

Mean changes in monthly headache days, medication days, and both migraine and headache intensity across $T 2$ to $T 5$ are summarised in Table 2. Normal distribution was confirmed for changes from baseline in headache days $(P=0.216, P=0.843, P=0.513$, and $P=0.323)$, medication days $(P=0.095, P=0.520$, $P=0.672$, and $P=0.090)$, migraine intensity $(P=0.083, P=0.433, P=0.209$, and $P>0.999)$, and headache intensity $(P=0.478, P=0.388, P=0.978$, and $P=0.238)$ for all periods $T 2$ to $T 5$.

The estimated marginal mean change in monthly headache days between baseline and T4 was $-3.3 \pm$ 0.8 days $(95 \%-\mathrm{Cl}-4.9--1.7)$ in the tDCS group and $-0.7 \pm 0.7$ days $(95 \%-\mathrm{Cl}-2.2-0.9)$ in the sham group; the difference between the groups was statistically significant $(P=0.041)$. However, changes in medication days, migraine intensity, and headache intensity did not differ between the groups during T4 ( $P=0.118, P=0.831$, and $P=0.454$, respectively).

Secondary endpoints evaluating the estimated marginal changes from baseline in the scores in the HADS-D, HADS-A, and MIDAS at the first and the second follow-up visit are summarised in Table 3 . The average delay between the baseline visit and the first follow-up visit was $50 \pm 8$ days; the second followup visit took place $218 \pm 64$ days after baseline visit.

Page 8 of 18 
We were unable to build a mixed effects model to analyse whether the stimulation had an influence on aura, because changes from baseline to $\mathrm{T} 2, \mathrm{~T} 3, \mathrm{~T} 4$, and $\mathrm{T} 5$ in days with an aura were not normally distributed $(P=0.013, P<0.001, P<0.001, P<0.001)$. Using an ANOVA (which is more robust to distributional deviations from normality) and including into the analysis only participants who had reported at least one aura during the whole study period, we did not find differences in the changes from baseline in the number of auras in $T 1, T 2, T 3, T 4$, and $T 5$ between sham and verum $\operatorname{tDCS}(P=0.135$, $\mathrm{P}=0.359, \mathrm{P}=0.797, \mathrm{P}=0.180$, and $\mathrm{P}=0.907$, respectively).

\section{Safety}

Participants were asked to note adverse events experienced during stimulation. Tingling was reported by eight patients in the sham group and six in the verum group $(P=0.680)$. Nausea occurred in one patient in the sham group and three in the verum group $(P=0.317)$. Pain was felt by five participants receiving sham stimulation and four participants in the anodal group $(P>0.999)$. Finally, fatigue was reported by four patients in the sham group and six in the verum group $(P=0.414)$.

In addition, in the sham group, one patient reported pain in their right ear, one patient recalled a migraine attack in close temporal relationship with stimulation, and one patient noted a depressed mood during stimulation. In the verum tDCS group, one patient reported a migraine attack to have occurred during stimulation, and one participant recalled temporary pain in the right half of his face during stimulation. No serious adverse event occurred.

\section{Discussion}

Preventive treatment with anodal tDCS over occipital cortex resulted in a significantly lower number of migraine days after a 28-day stimulation period. The difference between the groups was $1.9 \pm 0.7$ days. Even though the proportion of 50\%-responders was higher in the verum tDCS group than in the sham group; the difference was not significant. Yet, in our opinion, reducing an average of five migraine days per month by two days can be clinically meaningful.

The changes in migraine days in our study were smaller than in a previous study by Viganò and coworkers who also applied anodal stimulation the visual cortex(21). In EM, they reported a reduction in migraine days from 15 to eight per two months during an eight-week stimulation period; they performed two stimulation sessions per week. However, comparability is limited because in their study, no sham group had been included and statistical analysis was not corrected for baseline characteristics. Furthermore, they did not report how migraine days were distinguished from other headache types.

In the present study, the influence of tDCS on migraine days was not discernible during stimulation (T1) but set in belatedly. It seems that, at the group level, the tDCS effect is building up in the first months after the intervention, and reaches its the maximum at T4. Previous studies have also confirmed such a lagging effect but have reported varying delays between the baseline examination and measurable changes $(21,25)$. However, the moment in which the effect occurs does not seem to depend solely on the time passed since the start of the stimulation. The number of sessions may be more important as only repeated stimulations have been reported to induce sustained changes in cortical excitability. That is, changes in cortical excitability induced by one session of tDCS were found to last only several minutes(19), but tDCS repeated daily led to a summation of the stimulation effects on evoked potentials(48).

The low number of 50\%-responders is probably explained by a considerable inter-individual variability in response to $\operatorname{tDCS}(30,31)$. Thus, it would be helpful if therapeutic responses could be predicted. We suggest investigating in future studies whether improvement of habituation heralds therapeutic success; previous research had shown that that (i) in prophylactic treatment with topiramate, 
improvement of habituation correlates with therapeutic success and that (ii) tDCS of the visual cortex can improve habituation $(21,49)$.

Interestingly, we recorded a slight increase in migraine days in the sham group during T1 and T2 (see Figure 3). The reason for this finding is unknown. However, previous research indicates that, in clinical trials, the quality of entries in headache diaries ameliorates over time(51). Hence, an increasing quality of the entries during $\mathrm{T} 1$ and $\mathrm{T} 2$ might have led to the identification of an increasing number of headache days as migraine days. Consequently, we must suspect a systematic error that does not affect generalisability of the results because both groups were affected.

Although negative expectancies have been found to reduce the effect of tDCS(52), we do not believe that the paradoxical increase in migraine days in the sham group was due to participants being able to guess group allocation. This is because, first, the reported side-effects did not differ between the groups and, second, the impact on migraine did not differ between the groups during the stimulation period.

Every secondary endpoint analysed in this study was negative. We only found tendencies towards a stronger reduction of depressive symptoms in the verum tDCS group and towards differences in the MIDAS score between the two groups at FUP1 $(P=0.052)$. However, there were no changes in the total number of headache days, the number of medication days, the average intensity of migraine and headache days, as well as the scores of HADS-A. These findings suggest that tDCS reduces the number of migraine days but changes neither the attacks itself nor comorbidities.

While the MIDAS score did not differ significantly between the two groups at baseline, there was a trend towards higher values in the sham group $(\mathrm{P}=0.093)$. The reason for this finding is unclear. We suspect random fluctuation because, at the first follow-up visit, the two groups had approximated - the MIDAS score had decreased in the sham tDCS group and increased in the verum tDCS group. At the second follow-up visit (FUP2), we found a decrease in both groups, which indicates that, in absolute values, the MIDAS score was lower in the verum tDCS group. This latter finding is not unexpected, as the number of migraine days decreased after the stimulation period.

Contrasting with our results, previous studies using various tDCS protocols have reported a reduction of the number headache days, the number of medication days and pain intensity, and attack duration, and an increased quality of life in migraine patients(22-24, 26, 27, 36). Moreover, in these studies the beneficial effects occurred earlier than in our sample. Unlike ours, these studies had methodological shortcomings as the statistical models were not corrected for baseline characteristics. Finally, we cannot exclude that to some extent, our differing results may be attributable to the self-administration of the treatment, as minor deviations of the electrode position can impact current path, the induced electric field, and the concentration of the electric field under the active electrode.(33-35)

No between-group difference persisted four months post-treatment (T5), suggesting a fleeting stimulation effect; the average pain intensity of individual attacks was not influenced. The only study investigating the long-term effect of tDCS on migraine attacks found its influence subsiding slowly as well and detected no effect 12 weeks after stimulation(36). It is possible that tDCS temporarily defies the tendency towards an altered cortical excitability - yet, the tendency persists and prevails after some time. Thus, this technique may tackle the pathophysiological cascade of migraine attacks in an advanced stage, but not at its start.

This study did not raise safety concerns against tDCS as no severe adverse events occurred. The number of patients reporting side effects of the stimulation did not differ significantly between the groups, suggesting that patients probably could not distinguish verum stimulation from sham tDCS. 
These encouraging results emphasise that tDCS can be an effective alternative when pharmacological preventive treatment is contraindicated, ineffective or disliked by the patient. A favourable side effect profile may increase adherence as fear of intolerance often prevents patients from taking prophylactic treatment.(54) This view is supported by studies reporting adherence rates of the Cefaly device that largely exceed those of pharmacological treatment approaches $(55,56)$.

The strengths of this study are the very long follow-up period and the randomised, controlled, and blinded assessment of the intervention. In addition, the data suggest that treatment successes can be achieved when patients apply the stimulation independently at home.

Some limitations must be noted. The classification of individual attacks was based on the headache diary; non-migraine days were not classified. In personal interviews, more headache attacks might have been identified as migraine attacks. In addition, we did not correct for multiple testing. In order to prevent the increase of the overall type I error by multiple testing, we used a gatekeeping strategy; the secondary endpoints are thus to be considered exploratory. Finally, we also aimed to include chronic migraine patients in a second arm with a different stimulus paradigm. However, the study was halted due to recruitment issues.

\section{Conclusion}

A 28-day self-administered transcranial direct current stimulation protocol is safe and results in a significantly lower number of migraine days than sham stimulation over five months, especially at three and four months after the intervention. It might be an alternative or a complementary intervention to pharmacological treatments.

However, the self-administered anodal tDCS over the visual cortex, as applied in this study, has no immediate effects and does not lead to between-group differences extending beyond four months. Consequently, it would be of interest to investigate in future studies if longer stimulation periods are able to provide longer-lasting therapeutic benefit.

In addition, our data suggest that tDCS has no effect on headaches other than migraine or on comorbid anxiety or depressive symptoms.

\section{Classification of Evidence}

This study provides Class II evidence that anodal tDCS over the visual cortex in episodic migraine results in a significantly lower number of monthly migraine days.

\section{Funding}

HP was funded by the Werner Dessauer Stiftung. C.C.R. received support from the SNSF (grant no. 100019L_173248) and from the European Research Council (ERC) under the European Union's Horizon 2020 research and innovation program (grant agreement No 725355, BRAINCODES).

\section{Conflicts of interest}

Heiko Pohl received speaker fees from TEVA Pharmaceuticals and honoraria from Eli Lilly.

Jean Schoenen is Investigator/advisor for Amgen, Allergan, Eli-Lilly, Novartis, Teva, and has received speaker fees from Novartis and Teva.

Hans H. Jung was advisor for Alexion, Alnylam, CSL-Behring, Ipsen, Mitsubishi, and Sanofi-Aventis, and received speaker fees from Alexion, Alnylam and Sanofi-Aventis

Franz Riederer has received speaker honoraria from Burgerstein Foundation, Lilly, Teva and Novartis. 


\section{References}

1. Goadsby PJ, Holland PR, Martins-Oliveira M, Hoffmann J, Schankin C, Akerman S. Pathophysiology of Migraine: A Disorder of Sensory Processing. Physiol Rev. 2017;97(2):553-622.

2. Evans RW, Seifert T, Kailasam J, Mathew NT. The use of questions to determine the presence of photophobia and phonophobia during migraine. Headache. 2008;48(3):395-7.

3. Headache Classification Committee of the International Headache Society (IHS) The International Classification of Headache Disorders, 3rd edition. Cephalalgia. 2018;38(1):1-211.

4. Vanagaite J, Pareja JA, Storen O, White LR, Sand T, Stovner LJ. Light-induced discomfort and pain in migraine. Cephalalgia. 1997;17(7):733-41.

5. Vingen JV, Pareja JA, Storen O, White LR, Stovner LJ. Phonophobia in migraine. Cephalalgia. 1998;18(5):243-9.

6. Ozkul Y, Uckardes A. Median nerve somatosensory evoked potentials in migraine. European Journal of Neurology. 2002;9(3):227-32.

7. Schoenen J, Wang W, Albert A, Delwaide PJ. Potentiation instead of habituation characterizes visual evoked potentials in migraine patients between attacks. Eur J Neurol. 1995;2(2):115-22.

8. Ambrosini A, Rossi P, De Pasqua V, Pierelli F, Schoenen J. Lack of habituation causes high intensity dependence of auditory evoked cortical potentials in migraine. Brain : a journal of neurology. 2003;126(Pt 9):2009-15.

9. Valeriani M, de Tommaso M, Restuccia D, Le Pera D, Guido M, lannetti DG, et al. Reduced habituation to experimental pain in migraine patients: a $\mathrm{CO} 2$ laser evoked potential study. Pain. 2003;105(1):57-64.

10. Bohotin V, Fumal A, Vandenheede M, Bohotin C, Schoenen J. Excitability of visual V1-V2 and motor cortices to single transcranial magnetic stimuli in migraine: a reappraisal using a figure-of-eight coil. Cephalalgia. 2003;23(4):264-70.

11. Mulleners WM, Chronicle EP, Palmer JE, Koehler PJ, Vredeveld JW. Visual cortex excitability in migraine with and without aura. Headache. 2001;41(6):565-72.

12. Chen WT, Wang SJ, Fuh JL, Lin CP, Ko YC, Lin YY. Persistent ictal-like visual cortical excitability in chronic migraine. Pain. 2011;152(2):254-8.

13. Coppola G, Schoenen J. Cortical excitability in chronic migraine. Curr Pain Headache Rep. 2012;16(1):93-100.

14. Coppola G, Pierelli F, Schoenen J. Is the cerebral cortex hyperexcitable or hyperresponsive in migraine? Cephalalgia : an international journal of headache. 2007;27(12):1427-39.

15. Bindman L, Lippold OC, Redfearn JW. The Action of Brief Polarizing Currents on the Cerebral Cortex of the Rat (1) during Current Flow and (2) in the Production of Long-Lasting after-Effects. J Physiol. 1964;172:369-82.

16. Antal A, Nitsche MA, Paulus W. Transcranial direct current stimulation and the visual cortex. Brain Res Bull. 2006;68(6):459-63.

17. Accornero N, Li Voti P, La Riccia M, Gregori B. Visual evoked potentials modulation during direct current cortical polarization. Exp Brain Res. 2007;178(2):261-6.

18. Antal A, Kincses TZ, Nitsche MA, Bartfai O, Paulus W. Excitability changes induced in the human primary visual cortex by transcranial direct current stimulation: direct electrophysiological evidence. Invest Ophthalmol Vis Sci. 2004;45(2):702-7.

19. Nitsche MA, Paulus W. Excitability changes induced in the human motor cortex by weak transcranial direct current stimulation. J Physiol. 2000;527 Pt 3:633-9.

20. Tfelt-Hansen $P$, Olesen J. Taking the negative view of current migraine treatments: the unmet needs. CNS Drugs. 2012;26(5):375-82.

21. Vigano A, D'Elia TS, Sava SL, Auve M, De Pasqua V, Colosimo A, et al. Transcranial Direct Current Stimulation (tDCS) of the visual cortex: a proof-of-concept study based on interictal electrophysiological abnormalities in migraine. J Headache Pain. 2013;14:23.

22. Rocha S, Melo L, Boudoux C, Foerster A, Araujo D, Monte-Silva K. Transcranial direct current stimulation in the prophylactic treatment of migraine based on interictal visual cortex excitability abnormalities: A pilot randomized controlled trial. J Neurol Sci. 2015;349(1-2):33-9. 
23. Antal A, Kriener N, Lang N, Boros K, Paulus W. Cathodal transcranial direct current stimulation of the visual cortex in the prophylactic treatment of migraine. Cephalalgia. 2011;31(7):820-8.

24. Ahdab R, Mansour AG, Khazen G, El-Khoury C, Sabbouh TM, Salem M, et al. Cathodal Transcranial Direct Current Stimulation of the Occipital cortex in Episodic Migraine: A Randomized Sham-Controlled Crossover Study. J Clin Med. 2019;9(1).

25. Dasilva AF, Mendonca ME, Zaghi S, Lopes M, Dossantos MF, Spierings EL, et al. tDCS-induced analgesia and electrical fields in pain-related neural networks in chronic migraine. Headache. 2012;52(8):1283-95.

26. Andrade SM, de Brito Aranha REL, de Oliveira EA, de Mendonca C, Martins WKN, Alves NT, et al. Transcranial direct current stimulation over the primary motor vs prefrontal cortex in refractory chronic migraine: A pilot randomized controlled trial. J Neurol Sci. 2017;378:225-32.

27. Auvichayapat $P$, Janyacharoen T, Tiamkao S, Krisanaprakornkit T, Thinkhamrop B, Auvichayapat N. Transcranial Direct Current Stimulation on Prophylactic Treatment in Migraine Patients, an OpenLabel Pilot Study. Srinagarind Med J. 2012;27(1):49-57.

28. Baschi R, Sava SL, La Salvia V, De Pasqua V, Schoenen J, Magis D. EHMTI-0317. Transcranial direct current stimulation in chronic migraine: a pilot trial combining cathodal visual and anodal dlpfc stimulation. The Journal of Headache and Pain. 2014;15(S1).

29. Cortese F, Pierelli F, Bove I, Di Lorenzo C, Evangelista M, Perrotta A, et al. Anodal transcranial direct current stimulation over the left temporal pole restores normal visual evoked potential habituation in interictal migraineurs. J Headache Pain. 2017;18(1):70.

30. Wiethoff S, Hamada M, Rothwell JC. Variability in response to transcranial direct current stimulation of the motor cortex. Brain Stimul. 2014;7(3):468-75.

31. Lopez-Alonso V, Cheeran B, Rio-Rodriguez D, Fernandez-Del-Olmo M. Inter-individual variability in response to non-invasive brain stimulation paradigms. Brain Stimul. 2014;7(3):372-80.

32. Minen MT, Begasse De Dhaem O, Kroon Van Diest A, Powers S, Schwedt TJ, Lipton R, et al. Migraine and its psychiatric comorbidities. J Neurol Neurosurg Psychiatry. 2016;87(7):741-9.

33. Woods AJ, Bryant V, Sacchetti D, Gervits F, Hamilton R. Effects of Electrode Drift in Transcranial Direct Current Stimulation. Brain Stimul. 2015;8(3):515-9.

34. Bikson M, Datta A, Rahman A, Scaturro J. Electrode montages for tDCS and weak transcranial electrical stimulation: role of "return" electrode's position and size. Clin Neurophysiol. 2010;121(12):1976-8.

35. Kim JH, Kim DW, Chang WH, Kim YH, Kim K, Im CH. Inconsistent outcomes of transcranial direct current stimulation may originate from anatomical differences among individuals: electric field simulation using individual MRI data. Neuroscience letters. 2014;564:6-10.

36. Auvichayapat $P$, Janyacharoen $T$, Rotenberg A, Tiamkao S, Krisanaprakornkit $T$, Sinawat $S$, et al. Migraine prophylaxis by anodal transcranial direct current stimulation, a randomized, placebocontrolled trial. Journal of the Medical Association of Thailand = Chotmaihet thangphaet. 2012;95(8):1003-12.

37. Deutsche Migräne- und Kopfschmerzgesellschaft e.V. Kopfschmerzkalender [Available from: http://www.dmkg.de/files/dmkg.de/PDF-Dokumente/Kopfschmerzkalender_DEUTSCH_5.11.2019.pdf. 38. Tassorelli C, Diener HC, Dodick DW, Silberstein SD, Lipton RB, Ashina M, et al. Guidelines of the International Headache Society for controlled trials of preventive treatment of chronic migraine in adults. Cephalalgia. 2018;38(5):815-32.

39. Nitsche MA, Doemkes S, Karakose T, Antal A, Liebetanz D, Lang N, et al. Shaping the effects of transcranial direct current stimulation of the human motor cortex. J Neurophysiol. 2007;97(4):3109-17.

40. Opitz A, Legon W, Rowlands A, Bickel WK, Paulus W, Tyler WJ. Physiological observations validate finite element models for estimating subject-specific electric field distributions induced by transcranial magnetic stimulation of the human motor cortex. Neuroimage. 2013;81:253-64.

41. Makarov S, Horner M, Noetscher G. Brain and Human Body Modeling: Springer International Publishing; 2019.

42. Shajahan PM, Glabus MF, Gooding PA, Shah PJ, Ebmeier KP. Reduced cortical excitability in depression. Impaired post-exercise motor facilitation with transcranial magnetic stimulation. The British journal of psychiatry : the journal of mental science. 1999;174:449-54. 
43. Croarkin PE, Nakonezny PA, Lewis CP, Zaccariello MJ, Huxsahl JE, Husain MM, et al. Developmental aspects of cortical excitability and inhibition in depressed and healthy youth: an exploratory study. Front Hum Neurosci. 2014;8:669.

44. Concerto C, Lanza G, Cantone M, Pennisi M, Giordano D, Spampinato C, et al. Different patterns of cortical excitability in major depression and vascular depression: a transcranial magnetic stimulation study. BMC Psychiatry. 2013;13:300.

45. Salustri C, Tecchio F, Zappasodi F, Bevacqua G, Fontana M, Ercolani M, et al. Cortical excitability and rest activity properties in patients with depression. J Psychiatry Neurosci. 2007;32(4):259-66.

46. Westfall PH, Krishen A. Optimally weighted, fixed sequence and gatekeeper multiple testing procedures. Journal of Statistical Planning and Inference. 2001;99(1):25-40.

47. Gronseth GS, Cox J, Getchius TSD. Amendments to the 2011 American Academy of Neurology Clinical Practice Guideline Process Manual 2015 [Available from: https://webcache.googleusercontent.com/search?q=cache:_72YcmnVGroJ:https://www.aan.com/site assets/home-page/policy-and-guidelines/guidelines/about-

guidelines/15processmanualamendment_v607.pdf+\&cd=1\&hl=en\&ct=clnk\&gl=us\#5.

48. Alonzo A, Brassil J, Taylor JL, Martin D, Loo CK. Daily transcranial direct current stimulation (tDCS) leads to greater increases in cortical excitability than second daily transcranial direct current stimulation. Brain Stimul. 2012;5(3):208-13.

49. Di Clemente L, Puledda F, Biasiotta A, Vigano A, Vicenzini E, Truini A, et al. Topiramate modulates habituation in migraine: evidences from nociceptive responses elicited by laser evoked potentials. J Headache Pain. 2013;14:25.

50. Filmer HL, Ehrhardt SE, Bollmann S, Mattingley JB, Dux PE. Accounting for individual differences in the response to tDCS with baseline levels of neurochemical excitability. Cortex; a journal devoted to the study of the nervous system and behavior. 2019;115:324-34.

51. Hoppe A, Weidenhammer W, Wagenpfeil S, Melchart D, Linde K. Correlations of headache diary parameters, quality of life and disability scales. Headache. 2009;49(6):868-78.

52. Turi Z, Bjorkedal E, Gunkel L, Antal A, Paulus W, Mittner M. Evidence for Cognitive Placebo and Nocebo Effects in Healthy Individuals. Sci Rep. 2018;8(1):17443.

53. Stewart WF, Lipton RB, Dowson AJ, Sawyer J. Development and testing of the Migraine Disability Assessment (MIDAS) Questionnaire to assess headache-related disability. Neurology. 2001;56(6 Suppl 1):S20-8.

54. Gallagher RM, Kunkel R. Migraine medication attributes important for patient compliance: concerns about side effects may delay treatment. Headache. 2003;43(1):36-43.

55. Danno $D$, ligaya $M$, Imai $N$, Igarashi $H$, Takeshima $T$. The safety and preventive effects of a supraorbital transcutaneous stimulator in Japanese migraine patients. Sci Rep. 2019;9(1):9900.

56. Vikelis M, Dermitzakis EV, Spingos KC, Vasiliadis GG, Vlachos GS, Kararizou E. Clinical experience with transcutaneous supraorbital nerve stimulation in patients with refractory migraine or with migraine and intolerance to topiramate: a prospective exploratory clinical study. BMC Neurol. 2017;17(1):97. 
Table 1. Baseline characteristics of the participants; MIDAS - Migraine Disability Assessment, HADS-A - anxiety subscale of the hospital anxiety and depression scale, HADS-D - depression subscale of the hospital anxiety and depression scale

Sham tDCS

Characteristic

$(\mathrm{N}=12) \quad(\mathrm{N}=11) \quad \mathrm{P}$ value

Age - years

Female sex - no. (\%)

Average number of migraine days during baseline

Average number of all headache days during baseline

$\begin{array}{cc}34 \pm 10 & 41 \pm 15 \\ 12(100 \%) & 10(90.1 \%)\end{array}$

0.211

Average number of medication days during baseline

$6 \pm 3$

$5 \pm 2$

$8 \pm 2 \quad 7 \pm 3$

0.118

Average baseline intensity migraine days during baseline -1 to $10 / 10$

$6 \pm 3 \quad 4 \pm 3$

0.239

Average baseline intensity of headache days during baseline -1 to

$5 \pm 2$

$6 \pm 1$

0.192

$10 / 10$

Average baseline total MIDAS score

Average baseline HADS-A score

Average baseline HADS-D score

\section{$5 \pm 1$}

$6 \pm 1$

$35 \pm 23 \quad 19 \pm 20$

0.199

0.178

Table 2. Estimated marginal mean changes from across T2 to T5 (i.e. four times 28-days); positive values imply an increase

\begin{tabular}{|c|c|c|c|}
\hline & Sham & tDCS & $P$ value \\
\hline Estimated marginal mean change from baseline in monthly migraine days & $0.2 \pm 0.4$ & $-1.7 \pm 0.5$ & 0.010 \\
\hline Estimated marginal mean change from baseline in monthly headache days & $-0.5 \pm 0.5$ & $-1.9 \pm 0.5$ & 0.102 \\
\hline $\begin{array}{l}\text { Estimated marginal mean change from baseline in the average pain level of } \\
\text { migraine attacks }\end{array}$ & $0.0 \pm 0.2$ & $-0.1 \pm 0.2$ & 0.711 \\
\hline $\begin{array}{l}\text { Estimated marginal mean change from baseline in the average pain level of } \\
\text { headache attacks }\end{array}$ & $0.0 \pm 0.2$ & $-0.3 \pm 0.2$ & 0.348 \\
\hline Estimated marginal mean change from baseline in monthly medication days & $-1.0 \pm 0.4$ & $-0.9 \pm 0.4$ & 0.957 \\
\hline
\end{tabular}

Table 3. Secondary endpoints evaluating estimated marginal mean changes from baseline at the first follow-up visit (FUP1, 50 \pm 8 days after the baseline visit) and the second follow-up visit (FUP2, $218 \pm 64$ days after the baseline visit); positive values indicate an increase; MIDAS - Migraine Disability Assessment, HADS-A - anxiety subscale of the hospital anxiety and depression scale, HADS-D - depression subscale of the hospital anxiety and depression scale

\begin{tabular}{l|l|cc|c} 
& & Sham & tDCS & P value \\
\hline FUP1 & Estimated marginal mean change from baseline in total MIDAS score & $-7.7 \pm 4.6$ & $5.9 \pm 4.1$ & 0.052 \\
& & $-0.5 \pm 0.7$ & $-0.6 \pm 0.7$ & 0.884 \\
& Estimated marginal mean change from baseline in HADS-A score & $-0.2 \pm 0.5$ & $-0.7 \pm 0.5$ & 0.535 \\
\hline & Estimated marginal mean change from baseline in HADS-D score & $-3.7 \pm 6.3$ & $-1.8 \pm 5.9$ & 0.838 \\
\hline \multirow{2}{*}{ FUP2 } & Estimated marginal mean change from baseline in total MIDAS score & $-1.0 \pm 1.5$ & $-1.8 \pm 1.7$ & 0.480 \\
& Estimated marginal mean change from baseline in HADS-A score & $1.2 \pm 0.7$ & $-0.9 \pm 0.7$ & 0.064
\end{tabular}




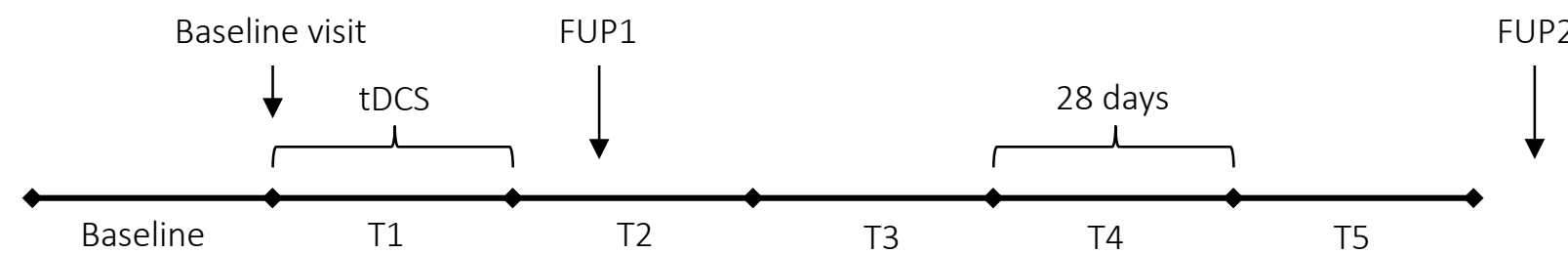

Figure 1. Overview of the study design; we subdivided the study period into six subsequent 28-day periods (baseline period, $\mathrm{T} 1, \mathrm{~T} 2, \mathrm{~T} 3, \mathrm{~T} 4$, and T5), during which participants recorded headache days in diaries; tDCS was applied during T1; all participants were completed the HADS and MIDAS in three presential visits (baseline visit, follow-up visit 1 - FUP1, and follow-up visit 2 FUP2); the baseline visit marked end of the baseline period and onset of treatment period T1; FUP 1 was scheduled shortly after the stimulation period (T1) and a FUP2 took place after T5 


\section{A Electrode montage}

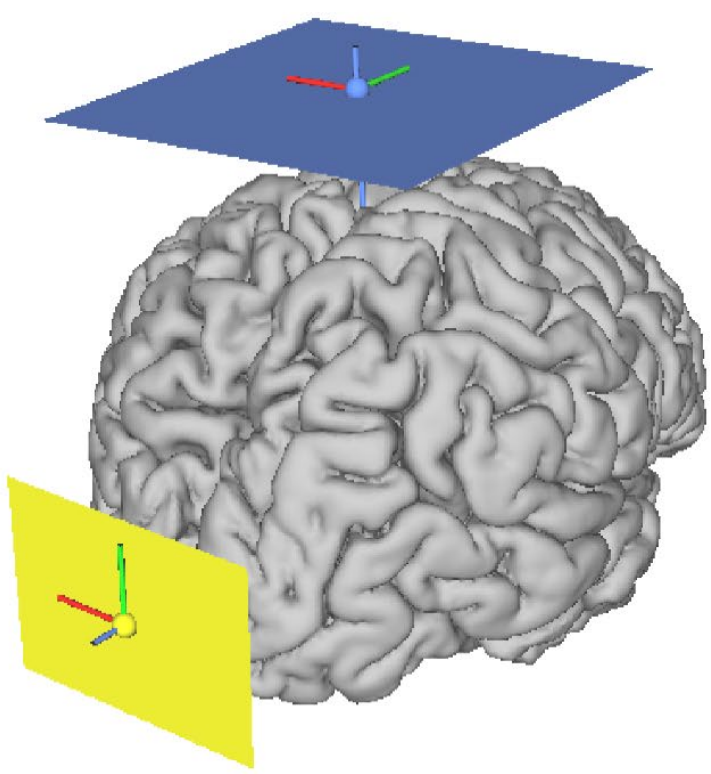

B Electric field simulation

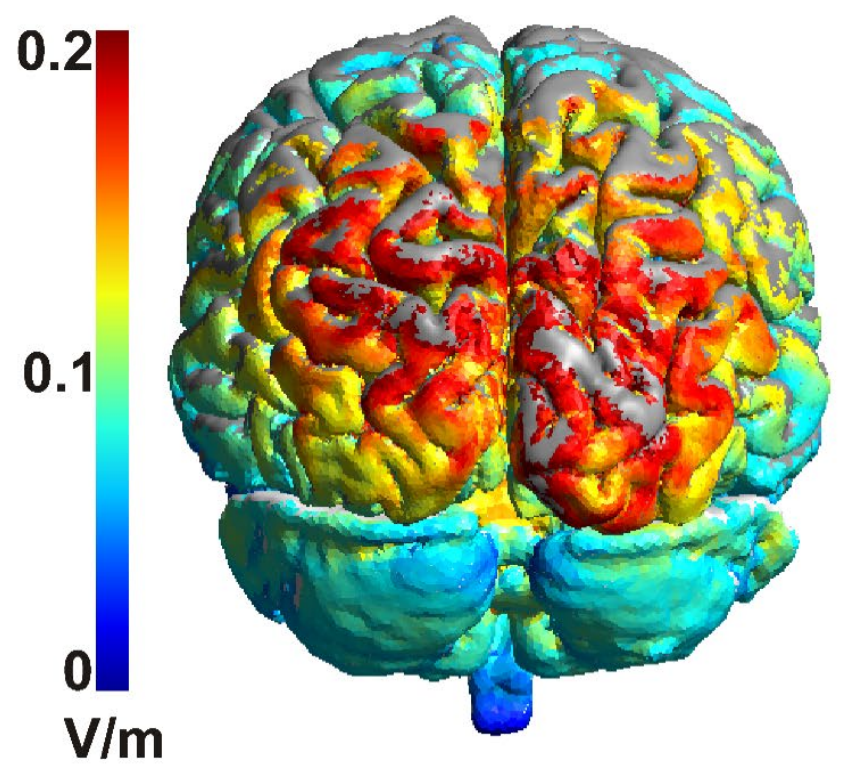

Figure 2 A The tDCS montage: the position of the active electrode over the visual cortex (size, $5 \times 7 \mathrm{~cm}^{2}$; current density 0.029 $\mathrm{mA} / \mathrm{cm} 2$ ) and of the reference electrode over the $\mathrm{Cz}$ (size, $10 \times 10 \mathrm{~cm}^{2}$; current density $\left.0.01 \mathrm{~mA} / \mathrm{cm}^{2}\right)$. B The predicted distribution of the normalized electric field. As expected, the predicted electric field is strongest under the active electrode placed over the visual cortex. 
Figure 3. Estimated marginal changes from baseline in migraine days for various time points calculated using mixed effect models; T1 refers to the 28-day stimulation period, and T2, T3, T4 and T5 refer to four subsequent 28-days follow-up periods; mean changes from baseline differ significantly at T4 $(P=0.033)$, there was a trend for significance at T3 $(P=0.081)$ but there was no significant difference in changes in migraine days between the two groups during stimulation (T1; $P=0.944), T 2$ $(P=0.497)$, and $T 5(P=0.631)$; error bars depict standard errors

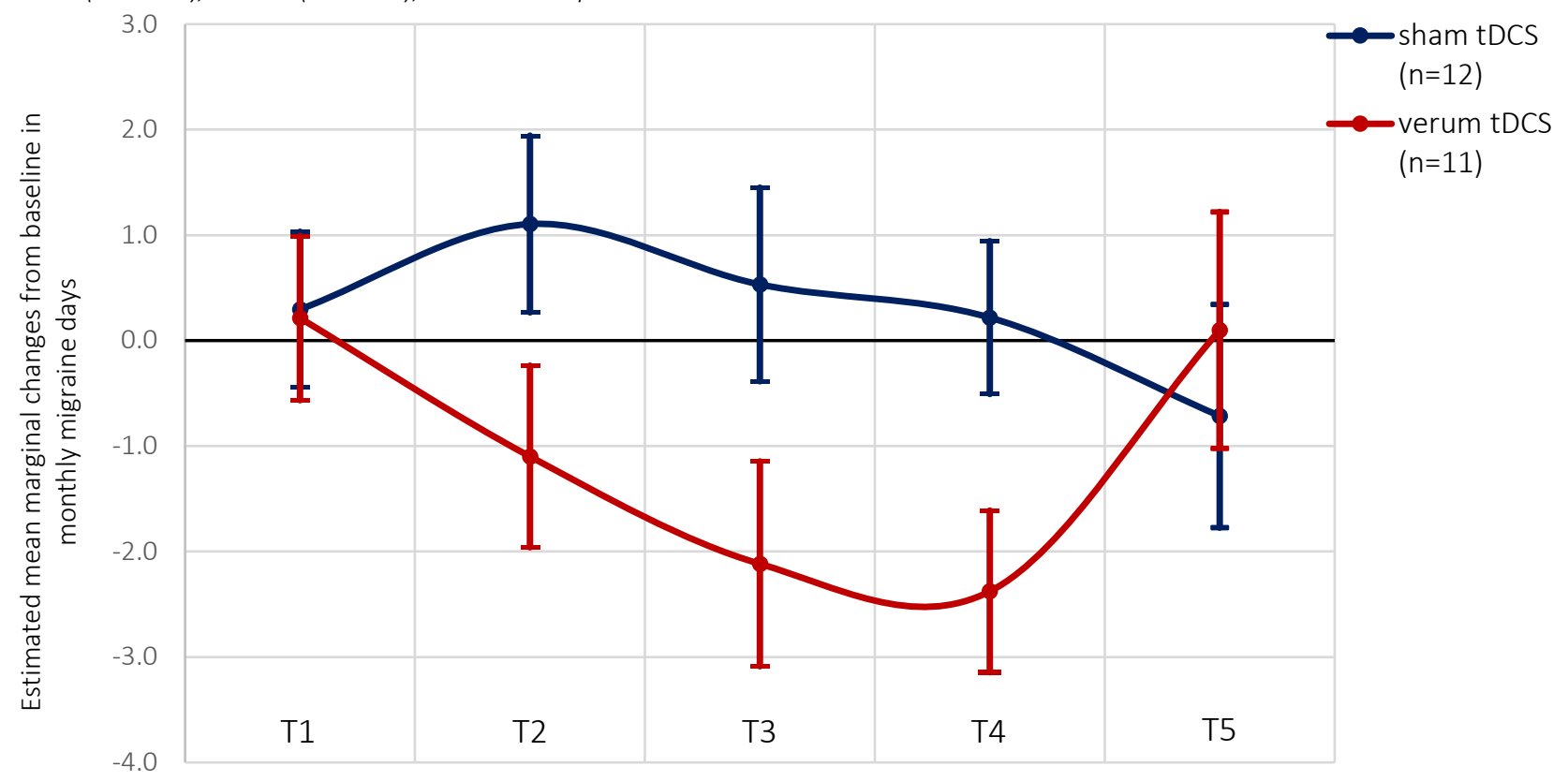

